\title{
A POLYNOMIAL TIME ALGORITHM FOR A DETERMINISTIC JOINT PRICING AND INVENTORY MODEL
}

\author{
Wilco van den Heuvel and Albert P.M. Wagelmans
}

\begin{tabular}{|l|l|}
\hline \multicolumn{2}{|l|}{ ERIM REPORT SERIES RESEARCH IN MANAGEMENT } \\
\hline ERIM Report Series reference number & ERS-2003-065-LIS \\
\hline Publication & 2003 \\
\hline Number of pages & 21 \\
\hline Email address corresponding author & wvandenheuvel@few.eur.nl \\
\hline Address & Erasmus Research Institute of Management (ERIM) \\
& Rotterdam School of Management / Faculteit Bedrijfskunde \\
& Rotterdam School of Economics / Faculteit \\
& Economische Wetenschappen \\
& Erasmus Universiteit Rotterdam \\
& P.O. Box 1738 \\
& 3000 DR Rotterdam, The Netherlands \\
& Phone: +31 10 408 1182 \\
& Fax: +31 10 408 9640 \\
& Email: info@erim.eur.nl \\
& Internet: www.erim.eur.nl \\
\hline
\end{tabular}

Bibliographic data and classifications of all the ERIM reports are also available on the ERIM website: www.erim.eur.nl 


\title{
ERASMUS RESEARCH INSTITUTE OF MANAGEMENT
}

\author{
REPORT SERIES \\ RESEARCH IN MANAGEMENT
}

\begin{tabular}{|c|c|c|}
\hline \multicolumn{3}{|c|}{ BIBLIOGRAPHIC DATA AND CLASSIFICATIONS } \\
\hline Abstract & \multicolumn{2}{|c|}{$\begin{array}{l}\text { In this paper we consider the uncapacitated economic lot-size model, where demand is a } \\
\text { deterministic function of price. In the model a single price need to be set for all periods. The } \\
\text { objective is to find an optimal price and ordering decisions simultaneously. In } 1973 \text { Kunreuther } \\
\text { and Schrage proposed an heuristic algorithm to solve this problem. The contribution of our paper } \\
\text { is twofold. First, we derive an exact algorithm to determine the optimal price and lot-sizing } \\
\text { decisions. Moreover, we show that our algorithm boils down to solving a number of lot-sizing } \\
\text { problems that is quadratic in the number of periods, i.e., the problem can be solved in polynomial } \\
\text { time. }\end{array}$} \\
\hline \multirow{3}{*}{$\begin{array}{l}\text { Library of Congress } \\
\text { Classification } \\
\text { (LCC) }\end{array}$} & $5001-6182$ & Business \\
\hline & $5201-5982$ & Business Science \\
\hline & HB 143 & Mathematical programming \\
\hline \multirow{3}{*}{$\begin{array}{l}\text { Journal of Economic } \\
\text { Literature } \\
\text { (JEL) }\end{array}$} & M & Business Administration and Business Economics \\
\hline & $\begin{array}{l}\text { M } 11 \\
\text { R } 4\end{array}$ & $\begin{array}{l}\text { Production Management } \\
\text { Transportation Systems }\end{array}$ \\
\hline & L 11 & Production, Pricing and Market Structure ... \\
\hline \multirow{3}{*}{$\begin{array}{l}\text { European Business Schools } \\
\text { Library Group } \\
\text { (EBSLG) }\end{array}$} & $85 \mathrm{~A}$ & Business General \\
\hline & $\begin{array}{l}260 \mathrm{~K} \\
240 \mathrm{~B}\end{array}$ & $\begin{array}{l}\text { Logistics } \\
\text { Information Systems Management }\end{array}$ \\
\hline & $255 \mathrm{~B}$ & Decision under constraints (programming) \\
\hline \multicolumn{3}{|c|}{ Gemeenschappelijke Onderwerpsontsluiting (GOO) } \\
\hline \multirow[t]{3}{*}{ Classification GOO } & 85.00 & Bedrijfskunde, Organisatiekunde: algemeen \\
\hline & $\begin{array}{l}85.34 \\
85.20 \\
\end{array}$ & $\begin{array}{l}\text { Logistiek management } \\
\text { Bestuurlijke informatie, informatieverzorging }\end{array}$ \\
\hline & 85.03 & Methoden en technieken, operations research \\
\hline \multirow[t]{3}{*}{ Keywords GOO } & \multicolumn{2}{|c|}{ Bedrijfskunde / Bedrijfseconomie } \\
\hline & \multicolumn{2}{|c|}{ Bedrijfsprocessen, logistiek, management informatiesystemen } \\
\hline & \multicolumn{2}{|c|}{ Productieplanning, prijsbeleid, series, voorraden, wiskundige programmering } \\
\hline Free keywords & \multicolumn{2}{|c|}{ Production; Pricing; Lot-sizing; Inventory } \\
\hline
\end{tabular}




\title{
A polynomial time algorithm for a deterministic joint pricing and inventory model
}

\author{
Wilco van den Heuvel ${ }^{a *}$ Albert P.M. Wagelmans ${ }^{a \dagger}$

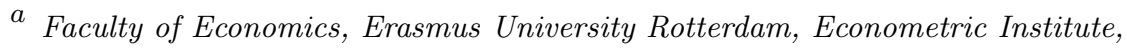 \\ P.O. Box 1738, 3000 DR Rotterdam, The Netherlands
}

\begin{abstract}
In this paper we consider the uncapacitated economic lot-size model, where demand is a deterministic function of price. In the model a single price need to be set for all periods. The objective is to find an optimal price and ordering decisions simultaneously. In 1973 Kunreuther and Schrage proposed an heuristic algorithm to solve this problem. The contribution of our paper is twofold. First, we derive an exact algorithm to determine the optimal price and lot-sizing decisions. Moreover, we show that our algorithm boils down to solving a number of lot-sizing problems that is quadratic in the number of periods, i.e., the problem can be solved in polynomial time.
\end{abstract}

Keywords: Production; Pricing; Lot-sizing; Inventory

\section{Introduction}

The demand that a manufacturer has to satisfy is usually created by activities of its marketing department. Instead of taking the marketing and production decisions more or less independently, it may be beneficial to integrate these decisions. This leads to models that are more complex than when we are only concerned with optimal production decisions. As an example, suppose that the selling price of the item still has to be set and that the demand functions are given for the periods under consideration. Then the planning problem consists of deciding simultaneously how high to set the price and how much to produce in each period such that the total profit is maximized.

In this paper we consider the uncapacitated economic lot-sizing problem (ELSP). This model can be described as follows. For a finite time horizon $T$, there is a known demand for a single

\footnotetext{
${ }^{*}$ Corresponding author. Tel.: +31-10-4081321, Email: wvandenheuvel@few.eur.nl

†Email: wagelmans@few.eur.nl
} 
product. This demand has to be satisfied each period by producing in this period or in previous periods, i.e., back-logging is not allowed. When production occurs in a period, a setup has to be made and marginal production costs per unit production are incurred. Finally, holding costs are incurred for carrying ending inventory from a period to the next one and it is assumed that all costs are non-negative. Now instead of assuming that demand is deterministic as in the classical ELSP, we assume that demand is a deterministic function of the price.

We see in the literature that two assumptions are made. First, one can assume that a different price can be set in each period. For instance, this is the case for a monopolist. Thomas (1970) showed that this problem can be solved in a similar way as the well-known algorithm proposed by Wagner and Whitin (1958). For instance, if demand is assumed to be linear in the price, then the algorithm requires $O\left(T^{2}\right)$ computation time. This means that this problem can still be solved efficiently.

Another assumption is that a single price must be set for all periods, i.e., $p_{t}=p$ for $t=1, \ldots, T$. For instance, this is the case if firms rely on sales from catalogues. In this case it may be rather expensive to communicate price changes to the customers. Kunreuther and Schrage (1973) propose an heuristic algorithm to solve this problem. However, they do not give any approximation and complexity results of their algorithm. In a more recent paper Gilbert (2000) considers the case with multiple items and production capacities. In this paper it is assumed that setup costs are negligible. The problem is solved iteratively by using the dual problem of the production scheduling problem. For an overview of literature on both continuous and discrete joint pricing-production decision-making we refer to Eliashberg and Steinberg (1993).

In this paper we propose an exact algorithm to solve the ELSP with deterministic demand functions and a constant price for all periods. We show that the problem can be solved in polynomial time. The remainder of the paper is organized as follows. In section 2 we give a mathematical formulation of the problem. In the next section we describe the heuristic algorithm proposed by Kunreuther and Schrage (1973). In section 4 we introduce an exact algorithm to solve the problem. In the last section we show that the running time of the algorithm is polynomial in the number of periods. The paper is ended up with a conclusion and we give some suggestions for future research.

\section{Problem description}

First, we will describe the classical ELSP in short. If we use the following notation

$T$ : model horizon

$D_{t}$ : demand in period $t$

$K_{t}:$ setup costs in period $t$ 
$c_{t}:$ unit production costs in period $t$

$h_{t}$ : unit holding costs in period $t$

$x_{t}$ : production quantity in period $t$

$I_{t}$ : ending inventory in period $t$

then the problem can be formulated as

$$
\begin{array}{rlr}
C(D)=\min & \sum_{t=1}^{T} K_{t} \delta\left(x_{t}\right)+c_{t} x_{t}+I_{t} h_{t} & \\
\text { s.t. } & I_{t}=I_{t-1}-D_{t}+x_{t} & t=1, \ldots, T \\
& x_{t}, I_{t} \geq 0, & t=1, \ldots, T \\
& I_{0}=0 &
\end{array}
$$

where

$$
\delta(x)= \begin{cases}0 & \text { for } x=0 \\ 1 & \text { for } x>0 .\end{cases}
$$

and $D$ is the demand vector of length $T$. The first set of constraints models that the ending inventory in period $t$ equals the ending inventory in period $t-1$ plus the amount produced in period $t$ minus demand in period $t$. The second set of constraints models that production and ending inventory are non-negative in each period. The above problem can be solved efficiently by dynamic programming. Wagner and Whitin (1958) proposed an $O\left(T^{2}\right)$ algorithm to solve the problem. Federgruen and Tzur (1991), Wagelmans et al. (1992) and Aggarwal and Park (1993) independently improved this result to $O(T \log T)$ for the general case and to $O(T)$ for certain interesting cases, such as stationary cost parameters.

Assume now that demand is not deterministic as in the ELSP, but demand is a deterministic function of the price and a single price for all periods needs to be set. Kunreuther and Schrage (1973) propose the demand function

$$
D_{t}(p)=\alpha_{t}+\beta_{t} d(p)
$$

where $d(p)$ is a differentiable non-increasing function. This means that demand will not increase, if the price increases. Note that the function $d(p)$ is independent of period $t$. If $d(p)=-p$ we have a linear demand curve. Now the objective is not to minimize the total costs as in the classical ELSP, but to maximize total profit. If we introduce the additional notation

$$
R_{t}(p)=p D_{t}(p) \text {, the revenue in period } t,
$$

then the objective can be formulated as

$$
\Pi(D(p))=\max _{p>0: D(p) \geq 0}\left\{\sum_{t=1}^{T} R_{t}(p)-C(D(p))\right\},
$$

where $D(p)$ corresponds to the vector $\left[D_{1}(p), \ldots, D_{T}(p)\right]$. 
Kunreuther and Schrage (1973) show that $C(D(p))$ is a concave piecewise linear function of the 'demand effect' $d(p)$. This can be seen in the following way. Let $S$ be the set of production periods (in increasing order) of a production plan. Furthermore, let $s(i)$ be the first production period after period $i$ and if period $i$ has no successor, then $s(i)=T+1$ by convention. Because of the zero-inventory property (see Wagner and Whitin (1958)), a production plan is completely determined by its production periods. Now the cost $S(d(p))$ of a production plan $S$ equals

$$
\begin{aligned}
S(d(p)) & =\sum_{i \in S} K_{i}+\sum_{i \in S} c_{i} \sum_{t=i}^{s(i)-1} D_{t}(p)+\sum_{i=1}^{T} \sum_{t=i+1}^{s(i)-1} h_{t-1} D_{i}(p) \\
& =\sum_{i \in S} K_{i}+\sum_{i \in S} c_{i} \sum_{t=i}^{s(i)-1}\left(\alpha_{t}+\beta_{t} d(p)\right)+\sum_{i=1}^{T} \sum_{t=i+1}^{s(i)-1} h_{t-1}\left(\alpha_{i}+\beta_{i} d(p)\right),
\end{aligned}
$$

which shows that $S(d(p))$ is a linear function of $d(p)$. Because $C(D(p))$ is the lower envelope of linear functions and the number of possible production plans is finite, $C(D(p))$ is a concave piecewise linear function of $d(p)$. This also means that each piece corresponds to a specific production plan $S$.

\section{Heuristic algorithm}

In this section we present the heuristic algorithm proposed by Kunreuther and Schrage (1973). The algorithm works as follows:

1. Set $i:=0$, start with some initial price $p_{i}$ and set $\Pi_{i}:=-\infty$.

2. Set $i:=i+1$.

3. Calculate the demand vector $D\left(p_{i-1}\right)$ and let $S_{i}$ be the optimal production plan corresponding to problem $C\left(D\left(p_{i-1}\right)\right)$

4. Calculate the price $p_{i}$ which maximizes the net profit $\Pi_{i}:=\sum_{t=1}^{T} R_{t}\left(p_{i}\right)-C\left(S_{i}\right)$

5. If $\Pi_{i}-\Pi_{i-1}>0$, then go to step 2 .

6. The terminating price is $p_{i}$ with corresponding net profit $\Pi_{i}$.

In the remainder of this paper we will call this the KS-procedure. In step 3 of the algorithm, we are looking for an optimal production plan given the demand vector $D\left(p_{i-1}\right)$, which boils down to solving an ELSP. In step 4 we are looking for an optimal price corresponding to a fixed production plan. If we have a relatively easy function $d(p)$ (for instance, $d(p)=-p$ ), the optimization problem may be relatively easy to solve (for instance, $\sum_{t=1}^{T} R_{t}(p)$ is quadratic for $d(p)=-p$ ). As in Kunreuther and Schrage (1973) we assume that the optimization problem in step 3 can be solved (either analytically or by a search procedure). 
Kunreuther and Schrage (1973) show that the above algorithm does not skip over any optimum on its way to the terminating price, i.e., if $p_{i-1}$ and $p_{i}$ are two successive prices generated by the KS-procedure, then $\sum_{t=1}^{T} R_{t}\left(p_{i}\right)-C\left(D\left(p_{i}\right)\right) \geq \sum_{t=1}^{T} R_{t}(p)-S(d(p))$ for all prices $p$ between $p_{i-1}$ and $p_{i}$ and all plans $S$. Thus, if we have a lower bound $p_{L}$ and an upperbound $p_{U}$ on the optimal price $p^{*}$ and we apply the KS-procedure with these values as initial prices resulting in terminating prices $p_{L}^{*}$ and $p_{U}^{*}$, respectively, then the optimal price $p^{*}$ satisfies the inequality $p_{L}^{*} \leq p^{*} \leq p_{U}^{*}$. So, if the terminating prices are equal, we have found the optimal price.

A graphical representation of the algorithm is shown in figure 1. In the figure it is assumed

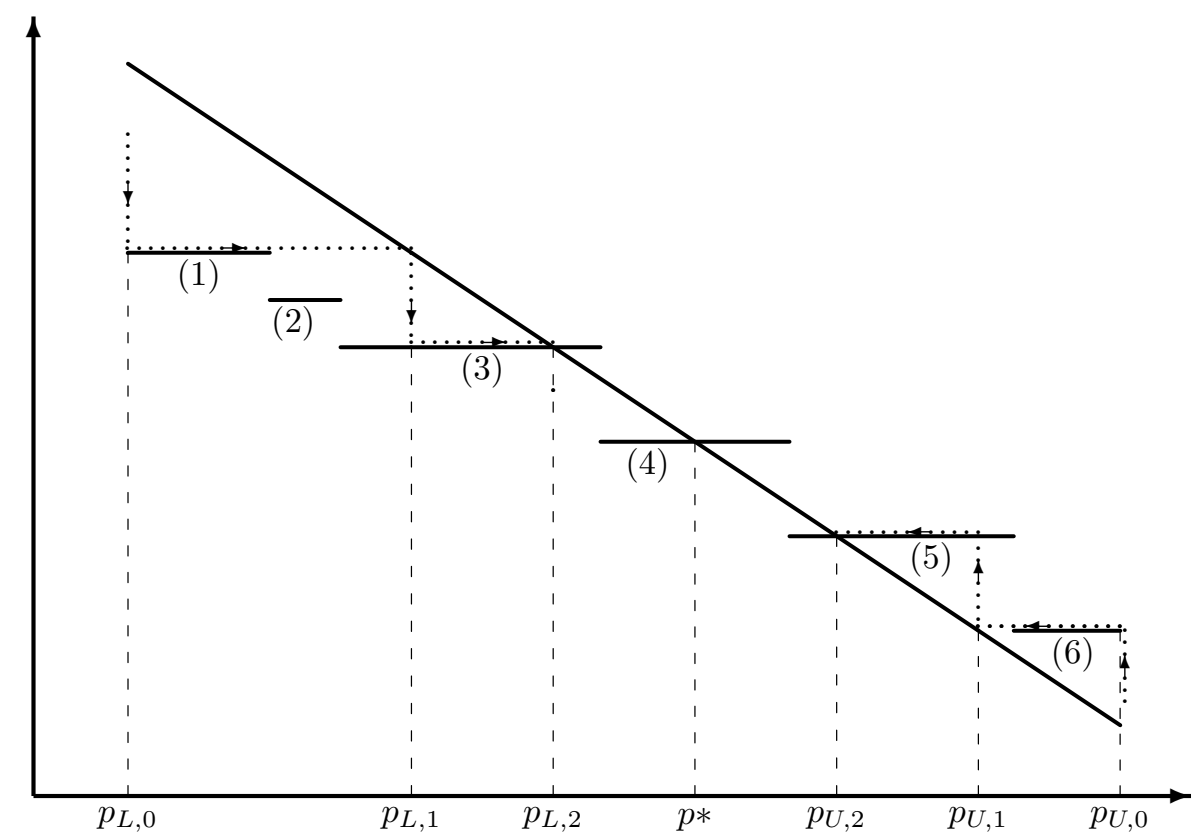

Figure 1: Heuristic algorithm

that $d(p)=-p$, so that $\sum_{t=1}^{T} R_{t}(p)$ is quadratic and $C(D(p))$ is a non-increasing concave piece wise linear function of $p$. The derivatives of the revenue and cost function are plotted against the price $p$. Because $d(p)=-p$ it follows immediately that the derivatives are a linear function and a piece wise constant function, respectively. The algorithm starts with some initial price $p_{L, 0}$. The marginal cost of the production plan corresponding to this price is line (1). In step 4 of the algorithm we are looking for the optimal price corresponding to this production plan. This optimal price occurs in the point where marginal revenue equals marginal cost $\left(p=p_{L, 1}\right)$. Now we are looking for the optimal production plan corresponding to the price $p=p_{L, 1}$, which is line (3). Note that the algorithm skips over line (2). It may be clear that there is no optimal solution in this part, because marginal revenue is larger than marginal cost. This means that it is not necessary 
to calculate all pieces of $C(D(p))$ explicitly. Now the optimal price corresponding to line $(3)$ is $p_{L, 2}$. At this point the algorithm terminates, because the optimal production plan corresponding to this price is again line (3).

In an analogous way we can find a terminating price starting with an upperbound for the optimal price $p^{*}$. Starting with price $p=p_{U, 0}$, we see that the algorithm also terminates in two iterations at price $p=p_{U, 2}$. So, essentially the KS-procedure consists of a local search with multiple starting points. In this example there is another local optimal price $p^{*}$ which satisfies $p_{L, 2}<p^{*}<p_{U, 2}$. If the net profit in $p^{*}$ is larger than in $p_{L, 2}$ and $p_{U, 2}$, then the KS-procedure will not find the optimal solution. Kunreuther and Schrage (1973) give an example for which the algorithm does not converge. A slight modification of that example shows that this may have major consequences for the net profit.

Example 1 Consider a three-period problem with $K_{1}=13, K_{2}=K_{3}=10, h_{t}=h=1$, $c_{t}=c=0$ and $D_{t}(p)=\alpha_{t}-\beta_{t} d(p)$ with $\alpha_{1}=25, \alpha_{2}=\alpha_{3}=22, \beta_{t}=\beta=12$ and $d(p)=-p$. If we start with $p_{L, 0}=0$, the algorithm terminates after one iteration at $p_{L, 1}=0.96$ with net profit $\Pi_{L}=-0.06$. Starting with $p_{U, 0}=2.08$ (the largest price for which demand is positive), the algorithm terminates after one iteration at $p_{U, 1}=1.46$ with net profit $\Pi_{U}=-2.56$. However, the optimal price equals $p^{*}=1.13$ with net profit $\Pi_{U}=0.44>\Pi_{L}>\Pi_{U}$. This example shows that the KS-procedure may fail to find a profitable solution if one does exist. In this case a firm may decide to produce not at all, while it is profitable to produce. We will consider this problem instance in more detail later. (Note: In this example all prices and profits have been rounded to nearest cent.)

\section{Exact algorithm}

In this section we present an exact algorithm to solve the ELSP with deterministic demand functions. The main idea of the exact algorithm is that the KS-procedure is restarted. Assume that the heuristic algorithm starts with $p_{L}<p_{U}$ and terminates with prices $p_{L}^{*}$ and $p_{U}^{*}$ which satisfy $p_{L} \leq p_{L}^{*}<p_{U}^{*} \leq p_{U}$. To restart the algorithm we need a suitable price $p$ that satisfies $p_{L}^{*}<\bar{p}<p_{U}^{*}$. In the exact algorithm we will find such a price by applying an idea often attributed to Eisner and Severance (see Eisner and Severance (1976)) on the function $C(D(p))$.

We will first explain how the Eisner Severance(ES)-method works. Assume we would like to perform a parametric analysis on an optimization problem

$$
\begin{gathered}
z(\lambda)=\frac{\min (c+\lambda d) x}{} \\
x \in X,
\end{gathered}
$$

where $X$ is a finite set. Here the vector of objective coefficients is a linear function of a parameter $\lambda$. 
Assume we have some algorithm A to solve this optimization problem for a fixed $\lambda$. It is wellknown that the objective function $z(\lambda)$ is a piece wise linear concave function of $\lambda$. Assume that we want to find all breakpoints in the interval $\left[\lambda_{L}, \lambda_{U}\right]$. This can be done in the following way. Apply algorithm $\mathrm{A}$ on the optimization problem with $\lambda=\lambda_{L}$ and $\lambda=\lambda_{U}$. Calculate the intersection point $\bar{\lambda}$ of the lines corresponding to $\lambda_{L}$ and $\lambda_{U}$, say $l_{L}(\lambda)$ and $l_{U}(\lambda)$. Perform algorithm $\mathrm{A}$ on the optimization problem with $\lambda=\bar{\lambda}$. If the objective value is equal to $l_{L}(\bar{\lambda})$ and $l_{U}(\bar{\lambda})$, there is no new line piece between $\lambda_{L}$ and $\lambda_{U}$. Otherwise, apply the above procedure recursevily with $\lambda_{L}:=\lambda_{L}$ and $\lambda_{U}:=\bar{\lambda}$ and with $\lambda_{L}:=\bar{\lambda}$ and $\lambda_{U}:=\lambda_{U}$.

An example of the ES-method is shown in figure 2. We see that the piece wise linear concave

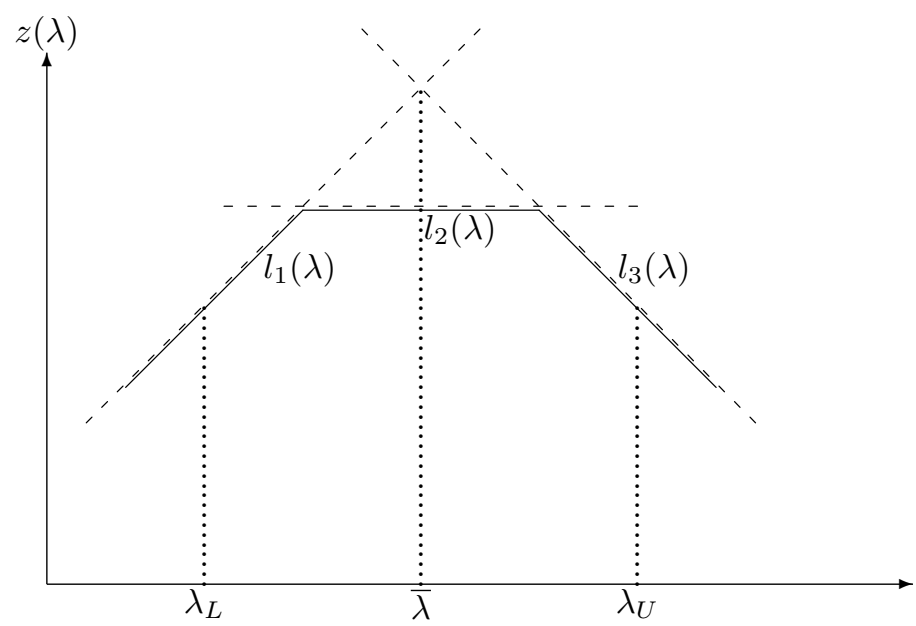

Figure 2: The Eisner Severance method

function consists of three line pieces: $l_{1}(\lambda), l_{2}(\lambda)$ and $l_{3}(\lambda)$. If we apply algorithm A with $\lambda=\lambda_{L}$ and $\lambda=\lambda_{U}$, the corresponding lines are $l_{1}(\lambda)$ and $l_{3}(\lambda)$, respectively. These lines intersect at $\lambda=\bar{\lambda}$. Applying algorithm A on the optimization problem with $\lambda=\bar{\lambda}$, we find line $l_{3}(\lambda)$. If we repeat this procedure with $\lambda_{L}:=\lambda_{L}$ and $\lambda_{U}:=\bar{\lambda}$ and with $\lambda_{L}:=\bar{\lambda}$ and $\lambda_{U}:=\lambda_{U}$, we will not find new line pieces and the ES-method terminates. If $B$ is the total number of breakpoints it is easy to show that algorithm A needs to be applied $2 B-1$ times. For more details we refer to Eisner and Severance (1976).

As already mentioned before, $C(D(p))$ is a piece wise linear function of $d(p)$ and each piece corresponds to a production plan. Assume now that the KS-procedure terminates with prices $p_{L}^{*}<p_{U}^{*}$. It follows from section 3 that in every iteration a new production plan is generated except for the last iteration. Because the interval $\left[p_{L}^{*}, p_{U}^{*}\right]$ has not been searched, it is possible that there are still production plans in $\left[p_{L}^{*}, p_{U}^{*}\right]$ that have a local or global maximum (see also figure 1 ). If $S_{L}^{*}$ and $S_{U}^{*}$ are the optimal production plans corresponding to $p_{L}^{*}$ and $p_{U}^{*}$, respectively, then by 
applying one iteration of the ES-method we can find the intersection point of the cost functions $S_{L}^{*}(d(p))$ and $S_{U}^{*}(d(p))$ in terms of $d(p)$, say in the point $d(p)=v$. Furthermore, because $d(p)$ is a continuous non-increasing function of $p$, there exists a $p$ with $p_{L}^{*}<p<p_{U}^{*}$ for which $d(p)=v$. Therefore we propose the following recursive algorithm.

Start the KS-procedure with $p_{L}<p_{U}$ and let $p_{L}^{*}$ and $p_{U}^{*}$ be the terminating prices If $p_{L}^{*}=p_{U}^{*}$ then

Stop; the optimal price is $p_{L}^{*}$

Else

Call $\operatorname{Optim}\left(p_{L}^{*}, p_{U}^{*}\right)$

End if

Function $\operatorname{Optim}\left(p_{L}, p_{U}\right)$

$$
\begin{aligned}
& \bar{p}=\mathrm{ES}\left(S_{L}, S_{U}\right) \\
& \text { If } \left.\left.S_{L}(d(\bar{p}))\right)=S_{U}(d(\bar{p}))\right) \text { then } \\
& \text { CheckOptima } \\
& \text { End function }
\end{aligned}
$$

Else

Perform the KS-procedure with $p=\bar{p}$ and let $\bar{p}^{*}$ be the terminating price If $\bar{p}^{*}>\bar{p}$, then

$$
\text { Call } \operatorname{Optim}\left(p_{L}, \bar{p}\right) ; \text { Call } \operatorname{Optim}\left(\bar{p}^{*}, p_{U}\right)
$$

Else

$$
\text { Call } \operatorname{Optim}\left(p_{L}, \bar{p}^{*}\right) ; \text { Call } \operatorname{Optim}\left(\bar{p}, p_{U}\right)
$$

End if

End if

End function

In the algorithm the function CheckOptima checks if the found optimum is larger than the previous found optimum (if any) and stores the corresponding price and production plan. The production plans $S_{L}$ and $S_{U}$ correspond to the prices $p_{L}$ and $p_{U}$, respectively. The function ES applies one iteration of the ES-method and finds a price $\bar{p}$ with the property $p_{L}<\bar{p}<p_{U}$ as described before. If there are no new production plans between $S_{L}$ and $S_{U}$, we can terminate the function Optim. If not, we apply the KS-procedure on the price $\bar{p}$. It is not clear beforehand, whether the terminating price $\bar{p}^{*}$ is larger of smaller than $\bar{p}$. If it is larger, then there may still be production plans between $p_{L}$ and $\bar{p}$ and between $\bar{p}^{*}$ and $p_{U}$. Therefore, we repeat the same procedure with starting prices $p_{L}$ and $\bar{p}$ and with prices $\bar{p}^{*}$ and $p_{U}$. If it is smaller, the procedure is repeated the other way around. In the following example we illustrate the exact algorithm. 
Example 2 Consider the same problem instance as in example 1. For this problem instance we have four possible production plans $S_{1}=\{1,2,3\}, S_{2}=\{1,2\}, S_{3}=\{1,3\}$ and $S_{4}=\{1\}$ with corresponding costs

$$
\begin{array}{cl}
S_{1}(p) & =33 \\
S_{2}(p)=S_{3}(p) & =45-12 p \\
S_{4}(p) & =79-36 p .
\end{array}
$$

The total revenue equals $R(p)=\sum_{t=1}^{T} R_{t}(p)=p(69-36 p)$. From example 1 we know that starting with $p_{L}=0$ the KS-procedure terminates at $p_{L}^{*}=0.96$ and starting with $p_{U}=2.08$ the KS-procedure terminates at $p_{U}^{*}=1.46$. The production plans corresponding to these prices are $S_{1}$ and $S_{4}$, respectively. For the marginal cost and marginal revenue functions we refer to figure 3 .

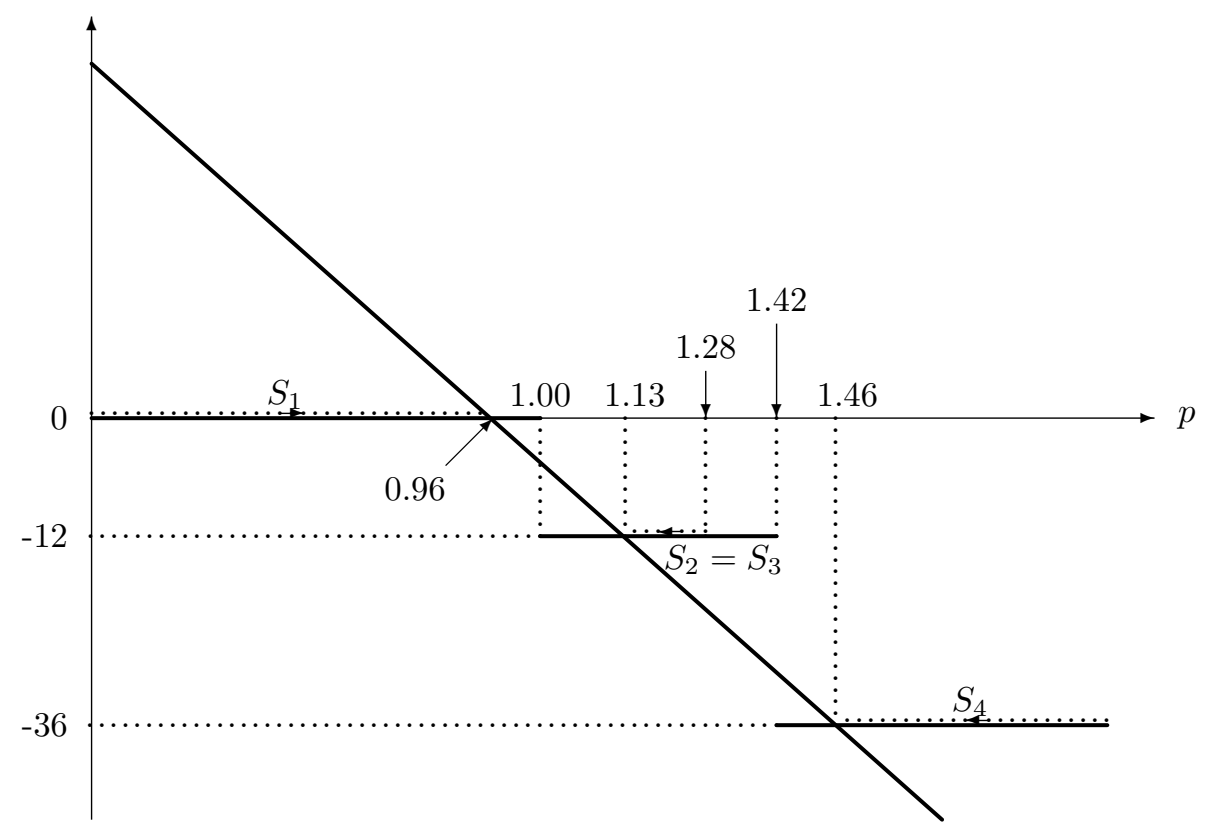

Figure 3: Marginal cost and marginal revenue functions

Whereas the KS-procedure stops, the exact algorithm continues and performs one step of the ES-method, i.e., the intersection point of $S_{1}(p)$ and $S_{4}(p)$ is calculated. These plans intersect in the point $p_{E S}=1.28$. Because $S_{1}(1.28) \neq S_{4}(1.28)$, the algorithm applies the KS-procedure with starting price $p_{E S}=1.28$. The optimal production plan corresponding to this price is $S_{2}$. The optimal price corresponding to $S_{2}$ is $p_{E S}^{*}=1.13$ and the KS-procedure terminates, because the optimal plan for this price is again $S_{2}$. (See also figure 3.)

Now it is possible that there are still optima in the interval $\left[p_{L}^{*}, p_{E S}^{*}\right]$ and $\left[p_{E S}, p_{U}^{*}\right]$. Therefore the procedure Optim is called again with prices $p_{L}^{*}, p_{E S}^{*}$ and $p_{E S}, p_{U}^{*}$. Starting this procedure with $p_{L}^{*}=0.96$ and $p_{E S}^{*}=1.13$, we calculate the intersection point of plans $S_{1}(p)$ and $S_{2}(p)$, which 
occurs at $p=1.00$. Because $S_{1}(1.00)=S_{2}(1.00)$, there are no new production plans between $p_{L}^{*}$ and $p_{E S}^{*}$ and the procedure terminates. Analogously, starting with $p_{E S}=1.28$ and $p_{U}^{*}=1.46$, the procedure terminates at price $p=1.42$.

Because the KS-procedure does not skip over any local optimum (see Kunreuther and Schrage (1973)), it must hold that $p_{L} \leq \bar{p}^{*} \leq p_{U}$. Furthermore, because the number of possible production plans is finite, the algorithm will always terminate. Moreover, because the whole interval $\left[p_{L}, p_{U}\right]$ is searched, the global optimum will be found. It is clear that the running time of the algorithm depends on the number of production plans. In the following section we will give an upper bound on the number of production plans that may be optimal for some value of $d(p)$.

\section{Time complexity of the algorithm}

In this section we will show that the time complexity of our algorithm is polynomial. To perform the ES-method we need to solve an ELSP and in every iteration of the KS-procedure we also need to solve an ELSP. We assume that step 4 of the KS-procedure requires less time then step 3, so that solving the ELSP takes the most time. Solving an ELSP requires $\mathrm{O}(T \log T)$ time in general. Because in every iteration of the KS-procedure a new production plan is generated (except for the last) and because the ES-method is applied to find a new production plan between two existing plans and terminates if such a plan is not found, the number of ELSP's to be solved is not larger than twice the number of production plans. Thus, if we are able to find a polynomial bound on the number of production plans, the algorithm runs in polynomial time. Because every production plan corresponds to a line piece in $C(D(p))$, it is sufficient to find a polynomial bound on the number of line pieces in $C(D(p))$ as a function of $d(p)$.

\subsection{The proof}

Theorem 1 The number of breakpoints of $C(D(p))$ as a function of $d(p)$ is at most $\frac{1}{2} T(T-1)$.

Note that the total number of different production plans is equal to $2^{T-1}$. We assume that in period 1 production takes place and in the remaining $T-1$ periods one may produce or not. We will prove theorem 1 by showing that a number of production plans will not occur in $C(D(p))$. Before we start the proof we give an example for model horizon $T=4$. If we denote the intercept of a line by $a$ and the slope by $-b$, then a line $l(p)$ can be represented by

$$
l(p)=a-b p
$$

and the intersection point of $l_{1}(p)$ and $l_{2}(p)$ occurs in

$$
p_{1,2}=\frac{a_{1}-a_{2}}{b_{1}-b_{2}} .
$$


It may be clear that if we have four lines $l_{1}(p), \ldots, l_{4}(p)$, then a necessary condition to have three breakpoints in the first quadrant is that if $a_{1}>a_{2}>a_{3}>a_{4}$ then $b_{1}>b_{2}>b_{3}>b_{4}$. If we have three breakpoints, then a picture of such a situation is as follows.

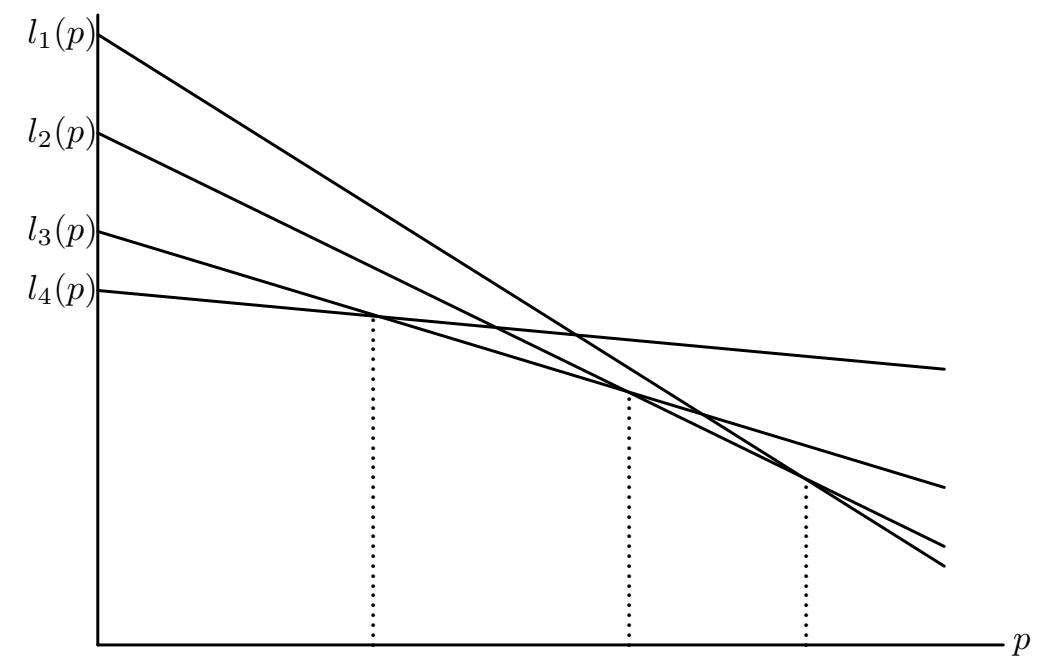

Figure 4: Example of 3 breakpoints

Example 3 Consider the ELSP with $T=4$ periods with demand in period $t$ according to $D_{t}(p)=\alpha_{t}-p \beta_{t}$. In this section a production plan is represented by a binary number. For instance the number 1010 corresponds to a production plan with production in period 1 and period 3. Now consider the following four production plans: 1010, 1011, 1110 and 1111 with the corresponding lines

$$
\begin{array}{ll}
1010: & l_{1}(p)=K_{1}+\left(\alpha_{1}+\alpha_{2}\right) c_{1}+K_{3}+\left(\alpha_{3}+\alpha_{4}\right) c_{3}-p\left(\left(\beta_{1}+\beta_{2}\right) c_{1}+\left(\beta_{3}+\beta_{4}\right) c_{3}\right) \\
1011: & l_{2}(p)=K_{1}+\left(\alpha_{1}+\alpha_{2}\right) c_{1}+K_{3}+\alpha_{3} c_{3}+K_{4}+\alpha_{4} c_{4}-p\left(\left(\beta_{1}+\beta_{2}\right) c_{1}+\beta_{3} c_{3}+\beta_{4} c_{4}\right) \\
1110: & l_{3}(p)=K_{1}+\alpha_{1} c_{1}+K_{2}+\alpha_{2} c_{2}+K_{3}+\left(\alpha_{3}+\alpha_{4}\right) c_{3}-p\left(\beta_{1} c_{1}+\beta_{2} c_{2}+\left(\beta_{3}+\beta_{4}\right) c_{3}\right) \\
1111: & l_{4}(p)=K_{1}+\alpha_{1} c_{1}+K_{2}+\alpha_{2} c_{2}+K_{3}+\alpha_{3} c_{3}+K_{4}+\alpha_{4} c_{4}-p\left(\beta_{1} c_{1}+\beta_{2} c_{2}+\beta_{3} c_{3}+\beta_{4} c_{4}\right)
\end{array}
$$

Note that

$$
\begin{aligned}
& a_{1}-a_{2}=a_{3}-a_{4}=\alpha_{4}\left(c_{3}-c_{4}\right)-K_{4} \\
& b_{1}-b_{2}=b_{3}-b_{4}=\beta_{4}\left(c_{3}-c_{4}\right) .
\end{aligned}
$$

Such a situation is illustrated in figure 5 .

Lemma 2 If we have four lines $l_{1}, \ldots, l_{4}$ with the properties $a_{1}-a_{2}=a_{3}-a_{4}, b_{1}-b_{2}=b_{3}-b_{4}$ and $b_{1} \neq b_{2}$, then we have not more than 2 breakpoints.

Proof It follows directly that we also have

$$
a_{1}-a_{3}=a_{2}-a_{4}
$$




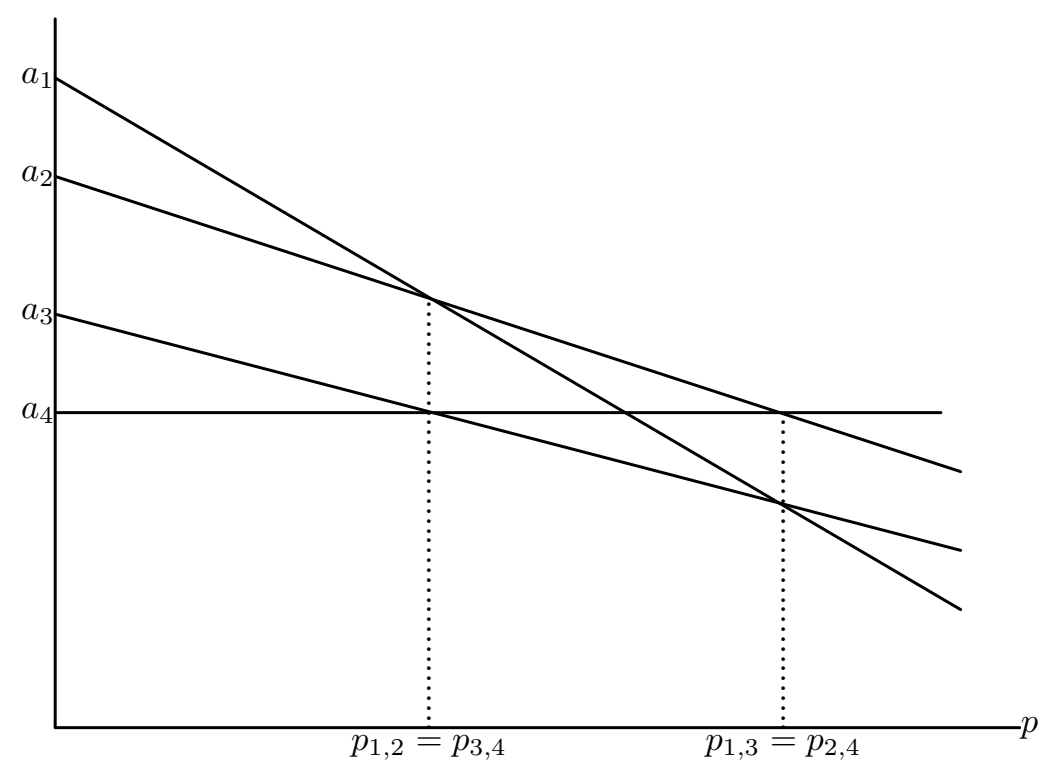

Figure 5: Example with $a_{1}>a_{2}>a_{3}$

and

$$
b_{1}-b_{2}=b_{3}-b_{4},
$$

implying that

$$
p_{1,2}=p_{3,4} \text { and } p_{1,3}=p_{2,4} .
$$

Assume without loss of generality that $a_{1} \geq a_{2}$, so that $a_{3} \geq a_{4}$. Now we have the following possibilities:

- $a_{1} \geq a_{2} \geq a_{3}$ :

It follows now that $a_{3} \geq a_{4}$, so that $a_{1} \geq a_{2} \geq a_{3} \geq a_{4}$. If we want to have three breakpoints, it must hold that $p_{1,2}<p_{2,3}<p_{3,4}$. But we know from (3) that $p_{1,2}=p_{3,4}$, implying that it is not possible to have three breakpoints.

- $a_{1} \geq a_{3} \geq a_{2}$ :

It follows now from (1) that $a_{2} \geq a_{4}$, so that $a_{1} \geq a_{3} \geq a_{2} \geq a_{4}$. If we want to have three breakpoints, it must hold that $p_{1,3}<p_{3,2}<p_{2,4}$. But we know from (3) that $p_{1,3}=p_{2,4}$, implying that it is not possible to have three breakpoints in this case.

- $a_{3} \geq a_{1} \geq a_{2}$ :

It follows now from (1) that it holds either that $a_{3} \geq a_{4} \geq a_{1} \geq a_{2}$ or that $a_{3} \geq a_{1} \geq$ $a_{4} \geq a_{2}$. In the first case to have three breakpoints, it must hold that $p_{3,4}<p_{4,1}<p_{1,2}$, which contradicts $(3)$. In the second case it must hold that $p_{3,1}<p_{1,4}<p_{4,2}$, which also contradicts (3). 
This means that in all cases it is not possible to have three breakpoints and that lines $l_{1}(p), \ldots, l_{4}(p)$ cannot have more than two breakpoints.

We can extend this proof to the case with $n$ pairs of lines.

Lemma 3 If we have $n \geq 2$ pairs of lines $l_{1}, \ldots, l_{2 n}$ with the properties $a_{2 i-1}-a_{2 i}=\triangle a$ and $b_{2 i-1}-b_{2 i}=\triangle b$ with $\triangle b \neq 0$ for $i=1, \ldots, n$, then we lose $n-1$ breakpoints.

Proof Assume that we have $n$ pairs of lines with the above property. The lines $l_{2 i-1}$ and $l_{2 i}$ correspond to pair $i$. Furthermore, we may assume without loss of generality that $\Delta b>0$, so that $l_{2 i-1}(p)>l_{2 i}(p)$ for $p<p^{*}$ and $l_{2 i-1}(p)<l_{2 i}(p)$ for $p>p^{*}$. Denote the lower envelope of the lines by $F_{n}(p)$, i.e.,

$$
F_{n}(p)=\min _{i=1, \ldots, 2 n} l_{i}(p) .
$$

It is clear that $F_{n}(p)$ has a breakpoint in $p^{*}=\triangle a / \triangle b$. Now we can distinguish the following two cases.

- There is exactly one pair that has an intersection point in $F_{n}\left(p^{*}\right)$. If we call this pair $i$, then

$$
i=\arg \min _{j=1, \ldots, n} l_{2 j-1}\left(p^{*}\right) .
$$

We will now show that every pair $j \neq i$ will contribute at most one line to $F_{n}(p)$. Assume that $p_{2 j, 2 i}<p^{*}$. Then we have that $b_{2 j}<b_{2 i}$ and the line $l_{2 j}(p)$ may contribute to $F_{n}(p)$. But then $l_{2 j-1}(p)$ will not contribute to $F_{n}(p)$, because

$$
b_{2 j-1}=\triangle b+b_{2 j}=b_{2 i-1}-b_{2 i}+b_{2 j}<b_{2 i-1}
$$

and

$$
l_{2 j-1}\left(p^{*}\right)>l_{2 i-1}\left(p^{*}\right),
$$

which implies that $l_{2 j-1}(p)>F_{n}(p)$ for $p>p^{*}$. The above situation is illustrated in figure 6 . Assume there is a pair $k$ with $p_{2 k-1,2 i-1}>p^{*}$. Then we have that $b_{2 k-1}>b_{2 i-1}$ and the line $l_{2 k}(p)$ may contribute to $F_{n}(p)$. But then $l_{2 k}(p)$ will not contribute to $F_{n}(p)$, because

$$
b_{2 k}=-\triangle b+b_{2 k-1}=-b_{2 i-1}+b_{2 i}+b_{2 k-1}>b_{2 i}
$$

and

$$
l_{2 k-1}\left(p^{*}\right)>l_{2 i-1}\left(p^{*}\right),
$$

which implies that $l_{2 k}(p)>F_{n}(p)$ for $p<p^{*}$. This situation is also illustrated in figure 6 . To summarize, there is exactly one pair (namely pair $i$ ) that contributes two line pieces to $F_{n}(p)$ and there are $n-1$ pairs that contribute at most one line piece to $F_{n}(p)$, so that at least $n-1$ breakpoints will not occur. 


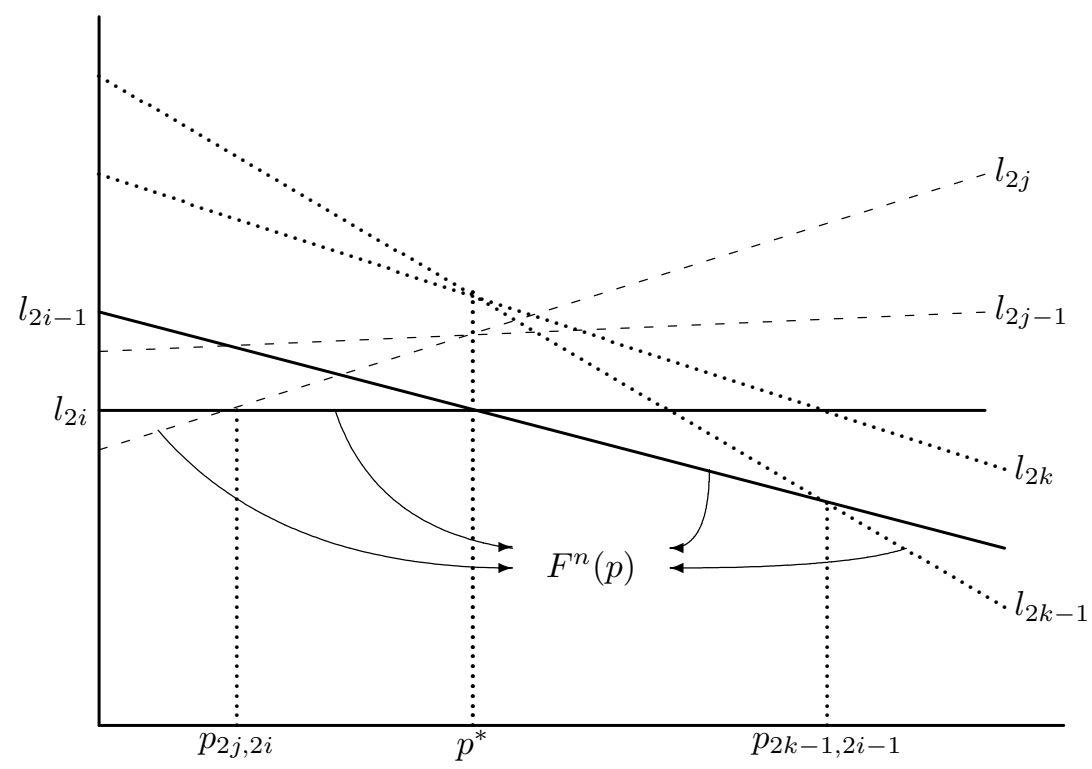

Figure 6: Example with $p_{2 j, 2 i}<p^{*}$ and $p_{2 k-1,2 i-1}>p^{*}$

- There are two or more pairs of lines that intersect in the point $F_{n}\left(p^{*}\right)$. In this case there are exactly two of theses pairs that contribute one line piece to $F_{n}(p)$ (see figure 7 ). One

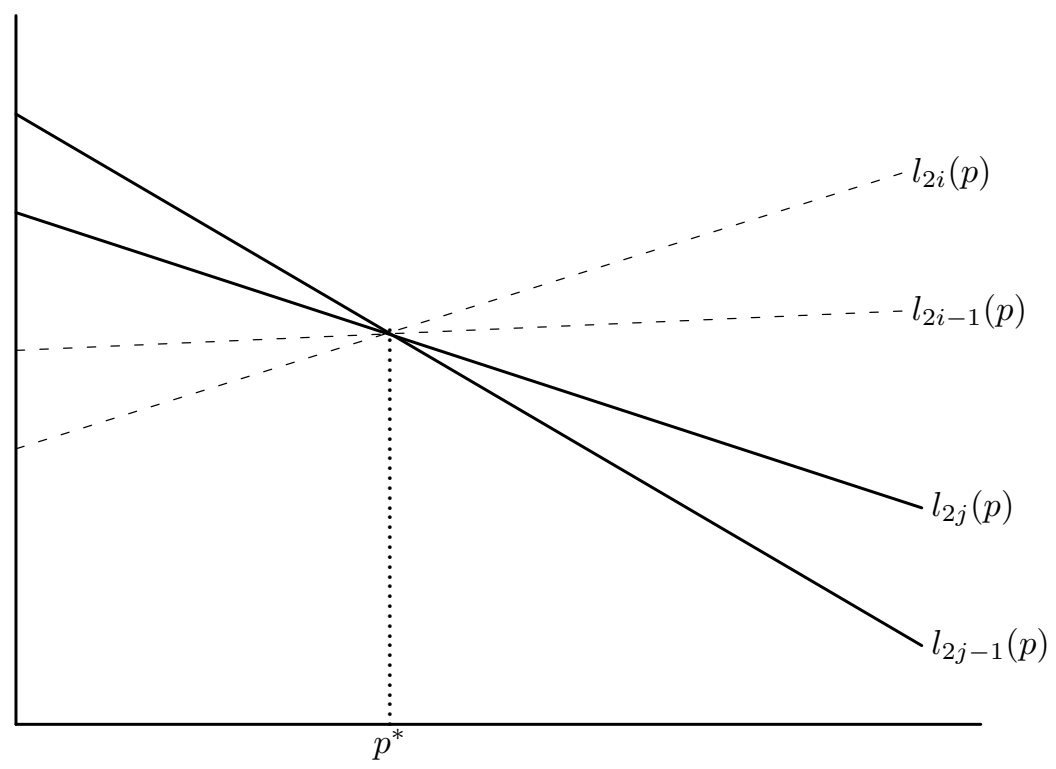

Figure 7: Two pairs of lines intersect in $p^{*}$

can prove in an analogous way as in the previous case that the pairs which do not intersect in $F_{n}\left(p^{*}\right)$, contribute at most one line piece to $F_{n}(p)$. Thus, if we have $k \geq 2$ pairs that 
intersect in $F_{n}\left(p^{*}\right)$, then $F_{n}(p)$ consists of at most $n-k+2$ line pieces, so that we lose at least $n+k-2>n-1$ breakpoints.

Thus, in both cases we lose at least $n-1$ breakpoints.

\section{Proof of theorem 1}

In the proof a production plan is represented by a binary number of length $T$. We assume that demand in period 1 is positive, so that a production plan is completely determined by a $T$-digit binary number starting with a 1 . Now consider the following pairs of production plans

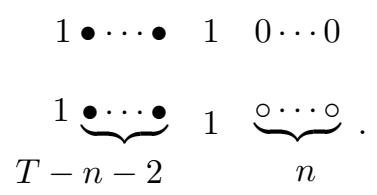

A •-sign in a production represents a period where production takes place in plan (4) if and only if production takes place in plan (5). Furthermore, in exactly one of the periods with a o-sign production takes place. For instance, if $T=5$, then the sequences

$$
\begin{array}{lll}
1 \cdot 1 & 00 \\
1 \bullet & 1 & 00
\end{array}
$$

represent two times two pairs of production plans

$\begin{array}{ll}10100 & 10100 \\ 10101 & 10110 \\ & \\ 11100 & 11100 \\ 11101 & 11110 .\end{array}$

We will now show that production pairs with identical 'tails' satisfy the properties of lemma 3. Let $S$ be the set of production periods (in increasing order) for a production plan. For instance with $T=5$ the production plan 10101 corresponds to $S=\{1,3,5\}$. Furthermore, if $i$ is an production period, then $s(i)$ is the successive production period and the successor of the last production period equals $T+1$ by convention. Now the cost $S(d(p))$ of a production plan $S$ as a function of $d(p)$ equals

$$
S(d(p))=\sum_{i \in S}\left(K_{i}+c_{i} \sum_{t=i}^{s(i)-1} D_{t}(p)\right)=\sum_{i \in S}\left(K_{i}+c_{i} \sum_{t=i}^{s(i)-1} \alpha_{t}\right)-d(p) \sum_{i \in S} c_{i} \sum_{t=i}^{s(i)-1} \beta_{t} .
$$

Note that there are no holding costs in (6). It can be shown (see for example Wagelmans et al. (1992)) that every ELSP can be rewritten as an ELSP without holding cost by making the substitution $c_{t}:=c_{t}+\sum_{i=t}^{T} h_{t}$. 
Assume now that we have a pair of production plans $S_{1}$ and $S_{2}$ according to (4) and (5), respectively, with period $m$ and $n(m<n)$ corresponding to the one but last and last production period in (5), i.e., $S_{1}=\left\{i_{1}, \ldots, i_{k}, m\right\}$ and $S_{2}=\left\{i_{1}, \ldots, i_{k}, m, n\right\}$. It follows from (6) that

$$
S_{2}(d(p))-S_{1}(d(p))=K_{n}+\left(c_{n}-c_{m}\right) \sum_{t=T-n+1}^{T} \alpha_{t}+p\left(c_{m}-c_{n}\right) \sum_{t=T-n+1}^{T} \beta_{t} .
$$

It follows directly from (7) that the difference in intercept and slope is independent of the sequence $i_{1}, \ldots, i_{k}$.

Now for a fixed $n \geq 1$ and a fixed production period in the last $n$ periods, there are $T-n-2$ places for the $\bullet$-sign, implying that we have $2^{T-n-2}$ pairs with the properties of lemma 3 . It follows from this lemma that we lose $2^{T-n-2}-1$ breakpoints. Because we have $n$ possibilities for the o-sign and $n$ ranges from 1 to $T-3$, we lose $\sum_{n=1}^{T-3} n\left(2^{T-n-2}-1\right)$ breakpoints in total. Furthermore, because the maximum number of breakpoints for model horizon $T$ equals $2^{T-1}-1$, the maximum number of breakpoints equals

$$
\begin{aligned}
& 2^{T-1}-1-\sum_{n=1}^{T-3} n\left(2^{T-n-2}-1\right)= \\
& 2^{T-1}-1+\frac{1}{2}(T-3)(T-2)-\sum_{n=1}^{T-3} n 2^{T-n-2}= \\
& 2^{T-1}-1+\frac{1}{2}(T-3)(T-2)-\left(-2(T-1)+2^{T-1}\right)= \\
& \frac{1}{2} T(T-1),
\end{aligned}
$$

where the second equality follows from lemma 4 (see appendix A).

As it follows from example 3 and figure 5 , if $l_{i}(p), l_{j}(p)$ and $l_{m}(p), l_{n}(p)$ are pairs of lines with the properties of lemma 3 , then the pairs $l_{i}(p), l_{m}(p)$ and $l_{j}(p), l_{j}(p)$ also have these properties. Call these pairs the complement pairs. Thus, to complete the proof we have to check that none of the pairs defined in (4) and (5) are complement pairs of each other. To see that this holds, note that the tails of the complement pairs (i.e., the production periods of the last $n+1$ periods) are identical for pairs constructed according to (4) and (5) with $n$ fixed. However, the tails of the production pairs defined in (4) and (5) are different. This means that the pairs can never be complements of each other. 


\subsection{An $\mathrm{O}\left(T^{2}\right)$ Example}

In this section we show that the bound on the number of optimal production plans in $C(D(p))$ in section 5.1 is tight. Consider the following problem instance. Let

$$
\begin{aligned}
& D_{t}(p)=D-p \quad \text { for } t=1, \ldots, T \\
& K_{t} \quad=(T-t+1) K \quad \text { for } t=1, \ldots, T \\
& c_{t} \quad=T^{T-t+1} \quad \text { for } t=1, \ldots, T \\
& h_{t}=0 \quad \text { for } t=1, \ldots, T \text {. }
\end{aligned}
$$

Again production plans are represented by binary numbers. We construct production plans in the following way. We start with a production plan with only a setup in period 1 . The next production production plan is constructed by adding a setup in period $T$ and the following production plans are constructed by moving this setup one period earlier. If this is not possible anymore, we start the procedure again by adding a setup in period $T$. Formally, if

$$
S_{i}=1 \bullet \cdots \bullet 010 \cdots 0
$$

is a production plan, then

$$
S_{i+1}=1 \bullet \cdots \bullet 100 \cdots 0
$$

is the next production plan, where $\bullet \cdots \bullet$ is the same sequence in both plans, and if

$$
S_{j}=1 \cdots 10 \cdots 00
$$

is a production plan, then

$$
S_{j+1}=1 \cdots 10 \cdots 01
$$

is the successive production plan. We will call this the 'in'-plans. For instance, the in-plans corresponding to $T=4$ are $1000,1001,1010,1100,1101,1110,1111$.

The main idea of the proof is as follows. If we only consider the 'in'-plans, we will show that these plans all contribute to the lower envelope. Then, if we consider the remaining production plans, the 'out'-plans (for instance, 1011 is an out-plan for $T=4$ ), we will show that they will not contribute to the lower envelope. Therefore, we will first show some properties of the production plans. Note that we can construct all production plans by binary counting, starting with the number $10 \cdots 0$ and ending with $11 \cdots 1$.

The first property is that the slopes of all plans are decreasing. Let $S_{1}$ be a production plan and $S_{2}$ be the successive production plan. Furthermore, let $k \leq T$ be the last non-production period and $n$ be the first production period before $k$ of plan $S_{1}$ (see the following picture).

$$
\begin{array}{lll} 
& n & k \\
S_{1}=1 \bullet \cdots \bullet & 10 \cdots 0 & 01 \cdots 1 \\
S_{2}=1 \bullet \cdots \bullet & 10 \cdots 0 & 10 \cdots 0
\end{array}
$$


If we denote the (negative) slope $b\left(S_{i}\right)$ of plan $S_{i}$ by

$$
b\left(S_{i}\right)=\sum_{i \in S} c_{i} \sum_{t=i}^{s(i)-1} 1,
$$

then we have that

$$
\begin{aligned}
& b\left(S_{1}\right)-b\left(S_{2}\right)= \\
& \sum_{i \in S_{1}} c_{i} \sum_{t=i}^{s(i)-1} 1-\sum_{i \in S_{2}} c_{i} \sum_{t=i}^{s(i)-1} 1= \\
& c_{n}+\sum_{t=k+1}^{T} c_{t}-c_{k}(T-k+1) \geq \\
& T \cdot T^{T-k+1}+\sum_{t=k+1}^{T} T^{T-k+1}-(T-k+1) T^{T-k+1} \geq \\
& \sum_{t=k+1}^{T} T^{T-k+1}>0 .
\end{aligned}
$$

Note that the third inequality holds, because $n \leq k-1$ which implies that $c_{n} \geq c_{k-1}=T c_{k}$ and the last inequality holds because $k \geq 2$ so that $T-1 \geq T-k+1$.

If $K$ is sufficient small, then we can show that the intercepts of the production plans (constructed by binary counting) are also decreasing. Recall that the intercept $a\left(S_{i}\right)$ of production plan equals

$$
a\left(S_{i}\right)=\sum_{i \in S_{i}}\left(K_{i}+c_{i} \sum_{t=i}^{s(i)-1} \alpha_{t}\right) .
$$

If we neglect the term $\sum_{i \in S_{i}} K_{i}$ and because demand is constant, we have

$$
a\left(S_{i}\right)=\sum_{i \in S_{i}} c_{i} \sum_{t=i}^{s(i)-1} D=D b\left(S_{i}\right) .
$$

Because $b\left(S_{i}\right)$ is decreasing, it is clear that the intercepts $a\left(S_{i}\right)$ are also decreasing.

For the intercepts of the in-plans we can prove a stronger result. Namely, the difference between the slopes of consecutive plans are decreasing, i.e., $b\left(S_{i}\right)-b\left(S_{i+1}\right)>b\left(S_{i+1}\right)-b\left(S_{i+2}\right)$. If $S_{i}, S_{i+1}$ and $S_{i+2}$ are of the following structure

$$
\begin{array}{lrll} 
& \multicolumn{2}{r}{n} & \multicolumn{1}{l}{k} \\
S_{i} & =1 \cdots 1 & 0 \cdots 000 & 10 \cdots 0 \\
S_{i+1} & =1 \cdots 1 & 0 \cdots 001 & 00 \cdots 0 \\
S_{i+2} & =1 \cdots 1 & 0 \cdots 010 & 00 \cdots 0
\end{array}
$$

then it holds that

$$
b\left(S_{i}\right)-b\left(S_{i+1}\right)=c_{n}-(T-k+1) c_{k}+(T-k+2) c_{k-1}
$$


and

$$
b\left(S_{i}\right)-2 b\left(S_{i+1}\right)+b\left(S_{i+2}\right)=(T-k+3) T^{2}-2(T-k+2) T+(T-k+1)>0
$$

for $T \geq 1$ and $T-k+1 \geq 0$, so that $b\left(S_{i}\right)-b\left(S_{i+1}\right)>b\left(S_{i+1}\right)-b\left(S_{i+2}\right)$. For other consecutive in-plans one can obtain the same result.

Now we consider the intersection points of two plans $S_{1}$ and $S_{2}$. It follows from 6 that if $S_{1}(p)=S_{2}(p)$, then

$$
p_{S_{1}, S_{2}}=D+\frac{\sum_{i \in S_{1}} K_{i}-\sum_{i \in S_{2}} K_{i}}{b\left(S_{1}\right)-b\left(S_{2}\right)} .
$$

Consider two consecutive in-plans $S_{i}$ and $S_{i+1}$. If we have a transition from plan $S_{i}$ to $S_{i+1}$ according to (13), then we have

$$
\sum_{j \in S_{i}} K_{j}-\sum_{j \in S_{i+1}} K_{j}=K_{k}-K_{k-1}=(T-k+1) K-(T-(k-1)+1) K=-K
$$

and if we have a transition according to

$$
\begin{array}{ll}
S_{i} & =1 \cdots 10 \cdots 00 \\
S_{i+1} & =1 \cdots 10 \cdots 01,
\end{array}
$$

then

$$
\sum_{j \in S_{i}} K_{j}-\sum_{j \in S_{i+1}} K_{j}=-K_{T}=-K
$$

This means that $\sum_{j \in S_{i}} K_{j}-\sum_{j \in S_{i+1}} K_{j}$ is constant. Because $b\left(S_{i}\right)-b\left(S_{i+1}\right)$ is decreasing, it follows from (14) that the intersection points are decreasing, which implies that all in-plans contribute to the lower envelope.

We will now show that the out-plans will not contribute to the lower envelope. Therefore, consider the following production plans

$$
\begin{array}{lrll} 
& \multicolumn{2}{r}{n} & \multicolumn{2}{l}{k} \\
S_{i} & =1 \cdots 1 & 0 \cdots 00 & 10 \cdots 0 \\
S_{i+1} & =1 \cdots 1 & 0 \cdots 00 & 10 \cdots 0 \\
S_{i+2} & =1 \cdots 1 & 0 \cdots 01 & 00 \cdots 0,
\end{array}
$$

where $S_{i+1}$ is a plan with at least one setup in the period(s) with a o-sign. Thus, $S_{i}$ is an in-plan with $S_{i+2}$ as a successor and $S_{i+1}$ is an out-plan. To show that $S_{i+1}$ does not contribute to the lower envelop, it is sufficient to show that $p_{S_{i+1}, S_{i+2}}>p_{S_{i}, S_{i+2}}$. We have that

$$
\sum_{i \in S_{i+1}} K_{i} \geq \sum_{i \in S_{i}} K_{i}+K_{m} \geq \sum_{i \in S_{i+2}} K_{i}
$$

for some $k<m \leq T$, because $K_{k}+K_{m} \geq K_{k-1}$. But then it follows from (14) that

$$
p_{S_{i}, S_{i+2}}<D \leq p_{S_{i+1}, S_{i+2}} .
$$


Because all out-plans have the same structure as $S_{i+1}$ in (16), only the in-plans contribute to the lower envelope.

To count the number of breakpoints of the lower envelope, we have to count the number of in-plans. We have exactly one in-plan with a setup in period 1, T-1 in-plans with 2 setups, $T-2$ in-plans with 3 setups, etc. This means that the total number of line pieces equals

$$
1+\sum_{t=1, \ldots, T-1} t=1+\frac{1}{2} T(T-1)
$$

and the number of breakpoints equals $\frac{1}{2} T(T-1)$.

\section{Conclusion \& Future research}

In this paper we have presented an exact algorithm to solve the ELSP with deterministic demand functions. We improved the heuristic algorithm proposed by Kunreuther and Schrage (1973) by showing that the problem is a parametric analysis on the demand vector of the ELSP. Therefore we applied on step of the Eisner Severance method to restart the Kunreuther Schrage algorithm in cases of no convergence. Furthermore, we have shown that the running time of our algorithm is polynomial in the number of periods $T$ under certain conditions.

For future research one may consider the capacitated version of the ELSP, the so-called capacitated lot-sizing model (CLSM). This problem is NP-hard in general, but there are some special cases that can be solved in polynomial time. If we add the extension of deterministic demand functions to these problems, maybe they are still solvable in polynomial time. However, these problems are more difficult to solve, because the cost function in the capacitated case is not piecewise linear concave anymore, but the cost function is discontinuous in general.

\section{A Appendix}

Lemma 4 For $n \geq 1$ it holds that

$$
\sum_{i=1}^{n} i 2^{n+1-i}=-2(n+2)+2^{n+2}
$$

Proof We will prove lemma 4 by induction. It is easy to verify that both the left and right hand side of (17) equal 2 for $n=1$. Assume now that lemma 4 holds for some $n \geq 1$. We will show that it also holds for $n+1$. We have 


$$
\begin{aligned}
\sum_{i=1}^{n+1} i 2^{n+2-i} & =\sum_{i=1}^{n} i 2^{n+2-i}+(n+1) 2 \\
& =2 \sum_{i=1}^{n} i 2^{n+1-i}+2 n+2 \\
& =2\left(2^{n+2}-2 n-4\right)+2 n+2 \\
& =2^{n+3}-2(n+3),
\end{aligned}
$$

which completes the proof.

\section{References}

Aggarwal, A., and Park, J.K., 1993. Improved algorithms for economic lot-size problems. Operations Research 14, 549-571.

Eisner, M.J., Severance, D.G., 1976. Mathematical techniques for efficient record segmentation in large shared databases. Journal of the Association for Computing Machinery, 23, 619-635.

Eliashberg, J., Steinberg, R, 1993. Marketing-production joint decision making. In: Eliashberg, J., Lilien, G. (Eds.), Handbooks In Operations Research and Management: Marketing, Elsevier Science Publishers, Amsterdam, pp. 827-880.

Federgruen, A., Tzur, M., 1991. A simple forward algorithm to solve general dynamic lot sizing models with $n$ periods in $\mathrm{O}(n \log n)$ or $\mathrm{O}(n)$ time. Management Science 37, 909-925.

Gilbert, S.M., 2000. Coordination of pricing and multiple-period production across multiple constant priced goods. Management Science 46, 1602-1616,

Kunreuther, H., Schrage, L., 1973. Joint pricing and inventory decisions for constant priced items. Management Science 19, 732-738.

Thomas, J., 1970. Price production decisions with deterministic demand. Management Science $16,747-750$.

Wagelmans, A.P.M., Van Hoesel, C.P.M., Kolen, A., 1992. Economic lot sizing: An O $(n \log n)$ algorithm that runs in linear time in the Wagner Whitin case. Operations Research 40, 81458156.

Wagner, H.M., Whitin, T.M., 1958. Dynamic version of the economic lot size model. Management Science 5, 89-96. 


\section{Publications in the Report Series Research* in Management}

ERIM Research Program: "Business Processes, Logistics and Information Systems"

2003

Project Selection Directed By Intellectual Capital Scorecards

Hennie Daniels and Bram de Jonge

ERS-2003-001-LIS

http://hdl.handle.net/1765/265

Combining expert knowledge and databases for risk management

Hennie Daniels and Han van Dissel

ERS-2003-002-LIS

http://hdl.handle.net/1765/266

Recursive Approximation of the High Dimensional max Function

Ş. II. Birbil, S.-C. Fang, J.B.G. Frenk and S. Zhang

ERS-2003-003-LIS

http://hdl.handle.net/1765/267

Auctioning Bulk Mobile Messages

S.Meij, L-F.Pau, E.van Heck

ERS-2003-006-LIS

http://hdl.handle.net/1765/274

Induction of Ordinal Decision Trees: An MCDA Approach

Jan C. Bioch, Viara Popova

ERS-2003-008-LIS

http://hdl.handle.net/1765/271

A New Dantzig-Wolfe Reformulation And Branch-And-Price Algorithm For The Capacitated Lot Sizing Problem With Set Up Times

Zeger Degraeve, Raf Jans

ERS-2003-010-LIS

http://hdl.handle.net/1765/275

Reverse Logistics - a review of case studies

Marisa P. de Brito, Rommert Dekker, Simme D.P. Flapper

ERS-2003-012-LIS

http://hdl.handle.net/1765/277

Product Return Handling: decision-making and quantitative support

Marisa P. de Brito, M. (René) B. M. de Koster

ERS-2003-013-LIS

http://hdl.handle.net/1765/278

\footnotetext{
A complete overview of the ERIM Report Series Research in Management: $\underline{\text { http://www.erim.eur.nl }}$

ERIM Research Programs:

LIS Business Processes, Logistics and Information Systems

ORG Organizing for Performance

MKT Marketing

F\&A Finance and Accounting

STR Strategy and Entrepreneurship
} 
Managing Product Returns: The Role of Forecasting

Beril Toktay, Erwin A. van der Laan, Marisa P. de Brito

ERS-2003-023-LIS

http://hdl.handle.net/1765/316

Improved Lower Bounds For The Capacitated Lot Sizing Problem With Set Up Times

Zeger Degraeve, Raf Jans

ERS-2003-026-LIS

http://hdl.handle.net/1765/326

In Chains? Automotive Suppliers and Their Product Development Activities

Fredrik von Corswant, Finn Wynstra, Martin Wetzels

ERS-2003-027-LIS

http://hdl.handle.net/1765/363

Mathematical models for planning support

Leo G. Kroon, Rob A. Zuidwijk

ERS-2003-032-LIS

http://hdl.handle.net/1765/332

How and why communications industry suppliers get "squeezed out" now, and the next phase

L-F Pau

ERS-2003-033-LIS

http://hdl.handle.net/1765/317

Financial Markets Analysis by Probabilistic Fuzzy Modelling

Jan van den Berg, Uzay Kaymak, Willem-Max van den Bergh

ERS-2003-036-LIS

http://hdl.handle.net/1765/323

WLAN Hot Spot services for the automotive and oil industries :a business analysis or : "Refuel the car with petrol and information , both ways at the gas station "

L-F Pau, M.H.P.Oremus

ERS-2003-039-LIS

http://hdl.handle.net/1765/318

A Lotting Method for Electronic Reverse Auctions

U. Kaymak, J.P. Verkade and H.A.B. te Braake

ERS-2003-042-LIS

http://hdl.handle.net/1765/337

Supply Chain Optimisation in Animal Husbandry

J.M. Bloemhof, C.M. Smeets, J.A.E.E. van Nunen

ERS-2003-043-LIS

http://hdl.handle.net/1765/353

A Framework for Reverse Logistics

Marisa P. de Brito and Rommert Dekker

ERS-2003-045-LIS

http://hdl.handle.net/1765/354

An assessment system for rating scientific journals in the field of ergonomics and human factors Jan Dul and Waldemar Karwowski

ERS-2003-048-LIS

http://hdl.handle.net/1765/432 
Circulation of Railway Rolling Stock: A Branch-and-Price Approach

Marc Peeters and Leo Kroon

ERS-2003-055-LIS

http://hdl.handle.net/1765/902

Emerging Multiple Issue e-Auctions

Jeffrey E. Teich, Hannele Wallenius, Jyrki Wallenius and Otto R. Koppius

ERS-2003-058-LIS

http://hdl.handle.net/1765/922

Inventory Management with product returns: the value of information

Marisa P. de Brito and E. A. van der Laan

ERS-2003-060-LIS

http://hdl.handle.net/1765/925

Promising Areas for Future Research on Reverse Logistics: an exploratory study

Marisa P. de Brito

ERS-2003-061-LIS

http://hdl.handle.net/1765/926 